\title{
Use of Nonlinear Frequency Modulated Signals for the Enhancement of Subharmonic Response from Contrast Microbubbles
}

\author{
MUHAMMAD ARIF*, MUHAMMAD ASIM**, AND FARIDA MEMON*** \\ RECEIVED ON 06.06.2016 ACCEPTED ON 16.08.2016
}

\begin{abstract}
Ultrasound imaging with the subharmonic component from contrast microbubbles provide improved CTR (Contrast-to-Tissue Ratio), however it is susceptible to the low amplitude of the subharmonic component. In this simulation study, NLFM (Nonlinear Frequency Modulated) signals are proposed in order to enhance the subharmonic response from microbubbles. NLFM signals having fractional bandwidths of 10, 20, and $40 \%$ with up and down sweeps were used as excitation. The performance of NLFM signals were compared with the reference tone-burst and LFM (Linear Frequency Modulated) signals. The results show that the ultrasound contrast microbubbles can produce subharmonic response which is dependent on the applied signal pressure and bandwidth. It is observed that the subharmonic component of the scattered NLFM signal is 3.2dB higher than the LFM signal, whereas it is $9 \mathrm{~dB}$ higher compared to the sinusoidal tone-burst signal. The results are also presented which show that the up and down sweeps NLFM signals performed better than the LFM signals at the same acoustic pressure and bandwidth.
\end{abstract}

Key Words: Nonlinear Chirp, Frequency Modulation, Contrast Agent, Microbubbles, Subharmonic Component, Ultrasound

\section{INTRODUCTION}

U

Trasound imaging using contrast microbubbles

has been widely used in diagnostic ultrasound

[1]. When microbubbles are insonated at low acoustic pressure, they can produce the fundamental frequency as well as second harmonic, ultra-harmonic and subharmonic frequencies. In ultrasound contrast imaging, these harmonic frequencies are used to improve the image contrast for blood detection from tissues [2]. Modern ultrasound scanners offer imaging with the second harmonic in order to enhance the image contrast with better axial resolution. However, the image quality can be degraded by the nonlinear second harmonic component produced by the tissue $[3,4]$.

On the other hand, ultrasound imaging with the subharmonic component can provide improved CTR as it is exclusively produced by the contrast microbubbles at low applied pressures [5]. The ultrasound subharmonic imaging is susceptible to the reduce axial resolution because the subharmonic component has a bandwidth which is one-half of the fundamental frequency. However, the subharmonic component will face less attenuation due

\footnotetext{
* Department of Biomedical Engineering, Mehran University of Engineering \& Technology, Jamshoro.

** Department of Electrical Engineering, Sukkur Institute of Business Administration, Sukkur.

*** Department of Electronic Engineering, Mehran University of Engineering \& Technology, Jamshoro.
}

Mehran University Research Journal of Engineering \& Technology, Volume 36, No. 1, January, 2017 [p-ISSN: 0254-7821, e-ISSN: 2413-7219] 
to the lower frequency and will improve the penetration depth $[6,7]$.

In the past decade, research works mainly focused to enhance the nonlinear response of contrast microbubbles. It was proved that the subharmonic response has a threshold value which depends on the applied acoustic pressure. This threshold has a minimum value if the applied signal frequency is double the bubble resonance frequency [8]. It has been observed that the subharmonic response is altered by the variations due to the ambient pressure [9]. It has been verified that the nonlinear subharmonic component is changed according to the applied frequency and signal shape [10]. It has been demonstrated that the nonlinear subharmonic component is produced due to the compression-only nature of the contrast microbubbles [11]. The applied low acoustic pressure on microbubbles will produce nonlinear harmonics with less microbubbles destruction. Moreover, it has the potential to use in many clinical applications such as real time estimation of non-invasive blood pressure [12], intravascular ultrasound subharmonic contrast imaging [13], contrast enhanced subharmonic imaging [14], and in-vivo perfusion estimation in kidneys using contrast microbubbles [15].

Many multiple excitation techniques are proposed in the past to increase the nonlinear response of contrast microbubbles at low acoustic pressure. Some of these famous techniques are the pulse inversion [16], pulse amplitude modulation [17], pulse inversion with amplitude modulation [18], microbubble radial modulation [19], and ultrasound CRCI (Chirp Reversal Contrast Imaging) [20]. All these techniques can provide improved SNR (Signalto-Noise Ratio) with better CTR and microbubble detection, however they can reduce the frame-rate of the system and susceptible to motion artifacts.
LFM chirp signals are long in duration and provide improved SNR without reducing the system frame-rate. However, pulse compression is required at the receiver to recover signal axial resolution. The power spectrum of an unweighted LFM chirp is approximately rectangular in shape and therefore produce higher sidelobes level after pulse compression. These higher sidelobes in the compressed signal can be reduce by applying a windowing function which can reduce the SNR [21]. NLFM are widely used in RADAR and SONAR communications to provide improved SNR [22]. NLFM signals are also suitable for ultrasound imaging [22] and ultrasonic non-destructive testing [23]. The NLFM chirps provide an alternative way to modify the chirp power spectrum into a desirable shape so that it can produce higher SNR and reduced sidelobes level after pulse compression [24,25].

The main aim of the present study is to use the NLFM signals in order to enhance the nonlinear response from contrast microbubbles. It is also to investigate the signal bandwidth effect on the subharmonic frequency component of the microbubble. The performance of the proposed NLFM signal is also compared to the toneburst and LFM (Linear Frequency Modulated) signals.

\section{THEORY AND DESIGN METHODS}

\subsection{Frequency Modulated Chirp Signals}

Frequency modulated signals have been used extensively in medical ultrasound scanners to get an improved image quality and better penetration depth [21].

The frequency modulated signal is defined as:

$$
x(t)=v(t) e^{j 2 \pi \int f_{i}(t) d t} \quad 0 \leq t \leq T
$$

where $v(t)$ is the signal envelop, $T$ is the chirps weeping time, and $f_{i}(t)$ is the instantaneous frequency of the chirp signal.

Mehran University Research Journal of Engineering \& Technology, Volume 36, No. 1, January, 2017 [p-ISSN: 0254-7821, e-ISSN: 2413-7219] 
For LFM signal, the instantaneous frequency is defined as [21]:

$f_{i}(t)=\frac{B}{T} t+\left(f_{c}-\frac{B}{2}\right)$

where $B$ is the signal bandwidth, and $f_{c}$ is the signal center frequency.

However, in the case of NLFM signal, the instantaneous frequency is defined as [25]:

$\mathrm{f}_{\mathrm{i}}(\mathrm{t})=\mathrm{f}_{\mathrm{c}}+\frac{\mathrm{B}}{\mathrm{T}}\left[\frac{\alpha \tan \left(\frac{2 \gamma \mathrm{T}}{\mathrm{T}}\right)}{\tan (\gamma)}+\frac{2(1-\alpha)}{\mathrm{T}} \mathrm{t}\right]$

The $\alpha$ and $\gamma$ values can be set to get the desired shape of the nonlinear instantaneous frequency curve.

\subsection{Excitation Signals}

The NLFM, LFM and tone-burst signals were used as an excitation in all simulations. The design parameters of the excitation signals and their values are shown in Table 1.

The $\alpha$ and $\gamma$ parameters of the NLFM signal can control the nonlinear curve of the instantaneous frequency function which can change the rectangular shape of the power spectrum and the $-3 \mathrm{~dB}$ bandwidth of the signal. Therefore, $\alpha$ and $\gamma$ parameters of the NLFM signal were carefully tuned to get the smooth NLFM power spectrum with equal $-3 \mathrm{~dB}$ bandwidth compared to the LFM signal.
For NLFM signal, a customized window function was used for amplitude tapering as designed in [25]. A Hann window was applied on both reference signals to get the smooth power spectra. In order to see the signal bandwidth effect to the subharmonic response, three fractional bandwidths 10, 20 and 40\% were used for NLFM and LFM signals. Fig. 1 is showing the NLFM, LFM, and tone burst excitation signals. The NLFM and LFM signals with a fractional bandwidth of $40 \%$ are shown in Fig. 1.

\section{SIMULATIONS}

The microbubble model of Marmottant et. al. [26] was programmed in the Matlab and used in all simulations. The model successfully defines the nonlinear response of contrast microbubbles. The model takes the effective surface tension as a function of bubble radius. The equation of bubble radial motion with effective surface tension is expressed as:

$\rho_{1}\left(R \ddot{R}+\frac{3}{2} \dot{R}^{2}\right)=\left(P_{0}+\frac{2 \sigma_{\omega}}{R_{0}}\right)\left(\frac{R}{R_{0}}\right)^{-3 \kappa}\left(1-\frac{3_{\kappa}}{c} \dot{R}\right)-P_{0}-\frac{2 \sigma(R)}{R}-\frac{4 \mu \dot{R}}{R}-\frac{4 \kappa_{s} \dot{R}}{R^{2}}-P_{a c}(t)$

The simulations were performed for SonoVue ${ }^{\circledR}$ (Sulphur Hexafluoride, Braco Research SA, Milan, Italy) which is a commercially available contrast agent. The bubble size distribution of SonoVue ${ }^{\circledR}$ is ranging from 0.7-10 $\mu \mathrm{m}[27]$, therefore, a bubble radius of $1.7 \mu \mathrm{m}$ was selected in the simulation. The parameters of the equation with simulation values for SonoVue $\AA$ are shown in Table-2.The microbubble effective surface tension $\sigma(R)$ with having elastic, buckling, and ruptured states are defined as:

TABLE 1. DESIGN PARAMETERS OF THE EXCITATION SIGNALS

\begin{tabular}{|c|c|c|c|c|}
\hline Excitation Signal Parameters & Symbol & NLFM & LFM & Tone-Burst \\
\hline Center Frequency & $\mathrm{f}_{\mathrm{c}}$ & $50 \mathrm{MHz}$ & $5.0 \mathrm{MHz}$ & $5.0 \mathrm{MHz}$ \\
\hline Time Duration & $\mathrm{T}$ & $10 \mu \mathrm{s}$ & $10 \mu \mathrm{s}$ & $10 \mu \mathrm{s}$ \\
\hline Frequency Bandwidth (\%) & $\mathrm{B}$ & $10,20,40$ & $10,20,40$ & 2 \\
\hline Gamma (NLFM) & $\gamma$ & 1.2 & - & - \\
\hline Alpha (NLFM) & $\alpha$ & 0.4 & - & - \\
\hline
\end{tabular}

Mehran University Research Journal of Engineering \& Technology, Volume 36, No. 1, January, 2017 [p-ISSN: 0254-7821, e-ISSN: 2413-7219] 


$$
\sigma(\mathrm{R})=\left\{\begin{array}{cc}
0 & \text { if } \mathrm{R} \leq \mathrm{R}_{\text {buckling }} \\
\chi\left(\frac{\mathrm{R}^{2}}{\mathrm{R}_{\text {buckling }}^{2}}-1\right. \\
\sigma_{\omega} & \text { if } \mathrm{R}_{\text {buckling }} \leq \mathrm{R} \leq \mathrm{R}_{\text {break-up }}
\end{array}\right.
$$

The buckling radius of the microbubble is defined as:

$$
\mathrm{R}_{\text {buckling }}=\mathrm{R}_{0}
$$

Whereas the radius of rupture microbubble is:

$$
\mathrm{R}_{\text {rupture }}=\mathrm{R}_{\text {buckling }} \sqrt{1+\frac{\sigma_{\omega}}{\chi}}
$$
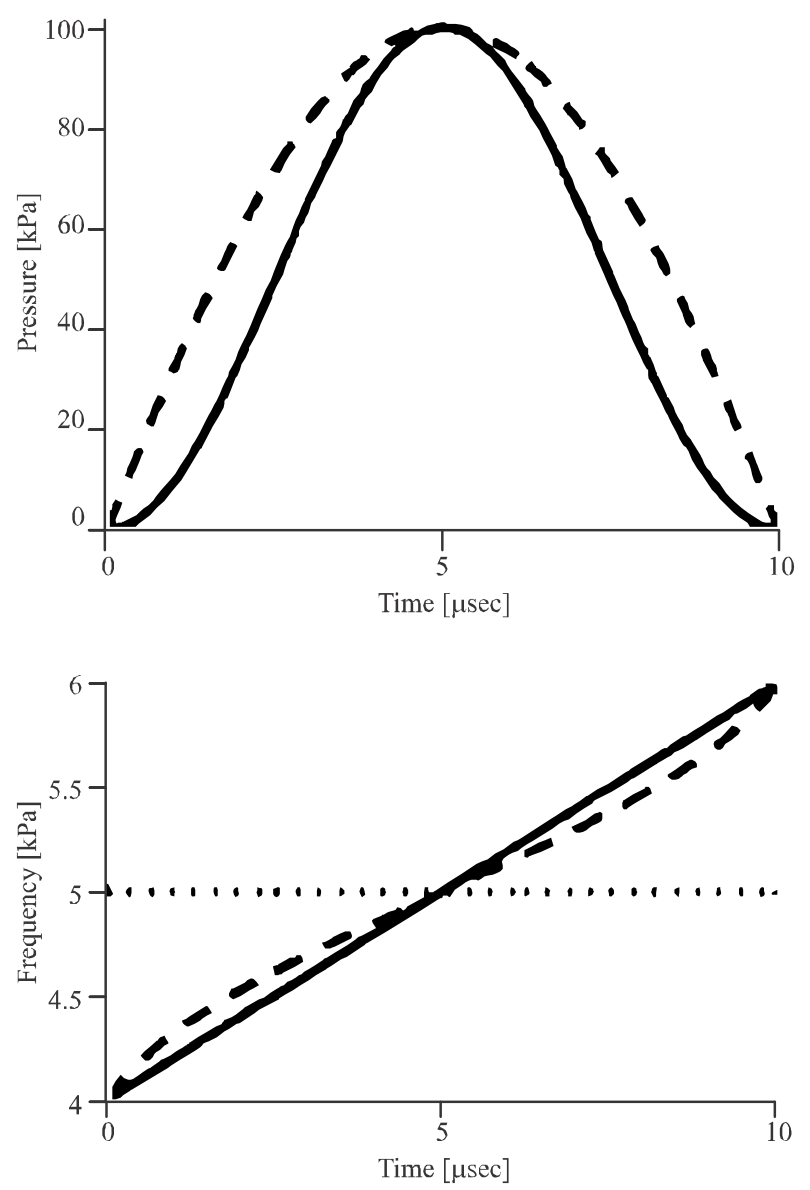

The pressure waveform scattered from the microbubble can be computed as [28]:

$$
P_{s}=\rho_{\mathrm{l}} \frac{R}{d}\left(2 \dot{R}^{2}+R \ddot{R}\right)
$$

Where $d$ is the distance of waveform measurement from the microbubble. The value of $d$ is normally set according to the focal length of the measuring transducer so that maximum scattered pressure from microbubble will be induced in the transducer. In this simulation study the measuring distance from microbubble was taken to $d=10$ $\mathrm{mm}$.
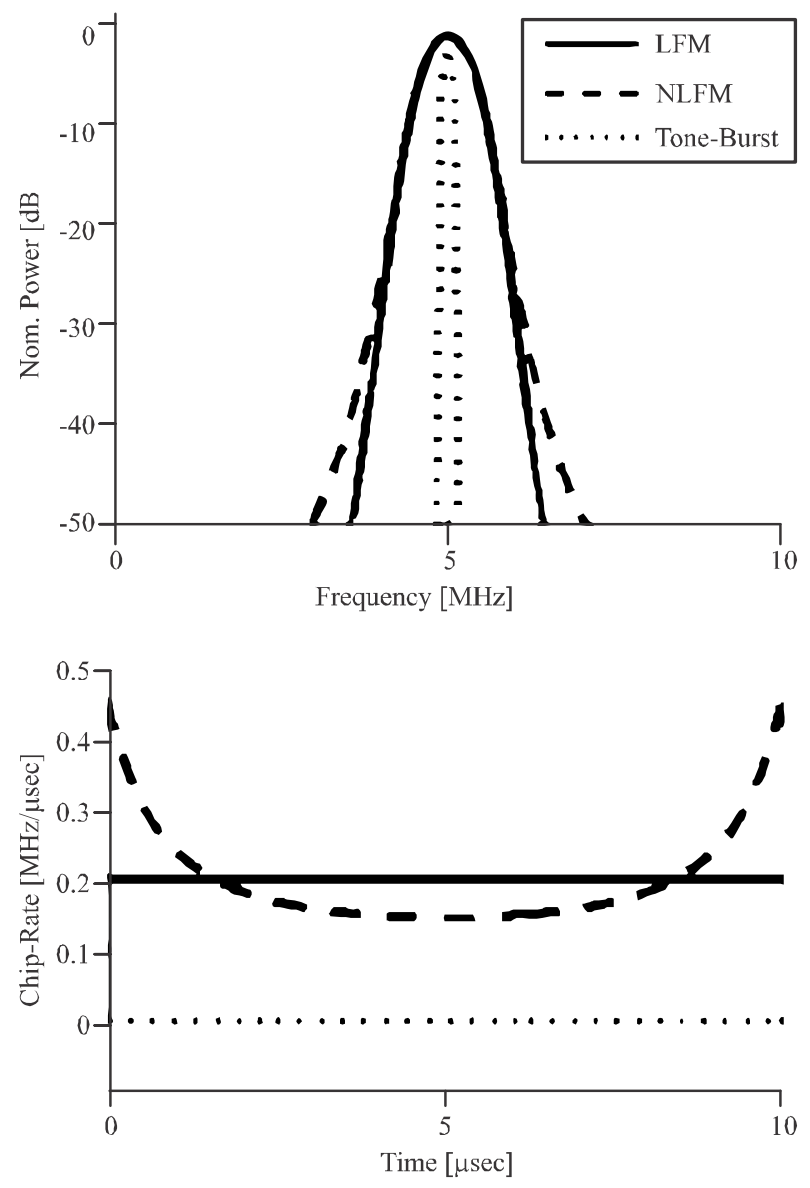

FIG. 1. (TOP-LEFT) SHOWING THE COMPARISON OF EXCITATION WAVEFORMS ENVELOPES, (TOP-RIGHT) THE SIGNALS SPECTRA, (BOTTOM-LEFT) INSTANTANEOUS FREQUENCIES, AND (BOTTOM-RIGHT) SHOWING THE CHIRP-RATE FUNCTIONS OF LFM, NLFM AND SINUSOIDAL TONE-BURST SIGNALS

Mehran University Research Journal of Engineering \& Technology, Volume 36, No. 1, January, 2017 [p-ISSN: 0254-7821, e-ISSN: 2413-7219] 
The symbols description and the values used in the simulation are shown in Table 2. The coated microbubble shell viscosity $\mu_{\mathrm{s}}$ and shell elasticity $\chi$ parameters values were taken from $[29,30]$.

TABLE 2. MODEL PARAMETERS AND THEIR VALUES USED IN THE SIMULATIONS

\begin{tabular}{|c|c|c|}
\hline Symbol & Parameter Definition & Value \\
\hline $\mathrm{R}_{0}$ & Bubble Radius at Equilibrium & $1.7 \times 10^{-6} \mathrm{~m}$ \\
\hline $\mathrm{R}$ & Instantaneous Bubble Radius & $\mathrm{m}$ \\
\hline$\dot{\mathrm{R}}$ & Velocity of the Bubble Wall & $\mathrm{m} / \mathrm{s}$ \\
\hline$\ddot{\mathrm{R}}$ & Acceleration of the Bubble Wall & $\mathrm{m} / \mathrm{s}^{2}$ \\
\hline Pac $(\mathrm{t})$ & Deriving Acoustic Pressure & $50 \times 10^{3} \mathrm{~Pa}$ \\
\hline$\rho_{1}$ & Density of the Liquid & $103 \mathrm{~kg} / \mathrm{m}^{3}$ \\
\hline $\mathrm{c}$ & Speed of Sound in the Liquid & $1.5 \times 10^{3} \mathrm{~m} / \mathrm{s}$ \\
\hline $\mathrm{P}_{0}$ & Ambient Pressure & $101.325 \times 10^{3} \mathrm{~Pa}$ \\
\hline$\kappa$ & Polytrophic Gas Exponent & 1.095 \\
\hline$\sigma_{\mathrm{w}}$ & Surface Tension Water & $72.8 \times 10^{-3} \mathrm{~N} / \mathrm{m}$ \\
\hline$\mu$ & Viscosity of the Liquid & $10^{-3} \mathrm{~Pa} . \mathrm{s}$ \\
\hline $\mathrm{K}_{\mathrm{S}}$ & Shell Viscosity & $4 \times 10^{-9} \mathrm{~kg} / \mathrm{s}$ \\
\hline$\chi$ & Shell Elasticity & $0.3 \mathrm{~N} / \mathrm{m}$ \\
\hline
\end{tabular}

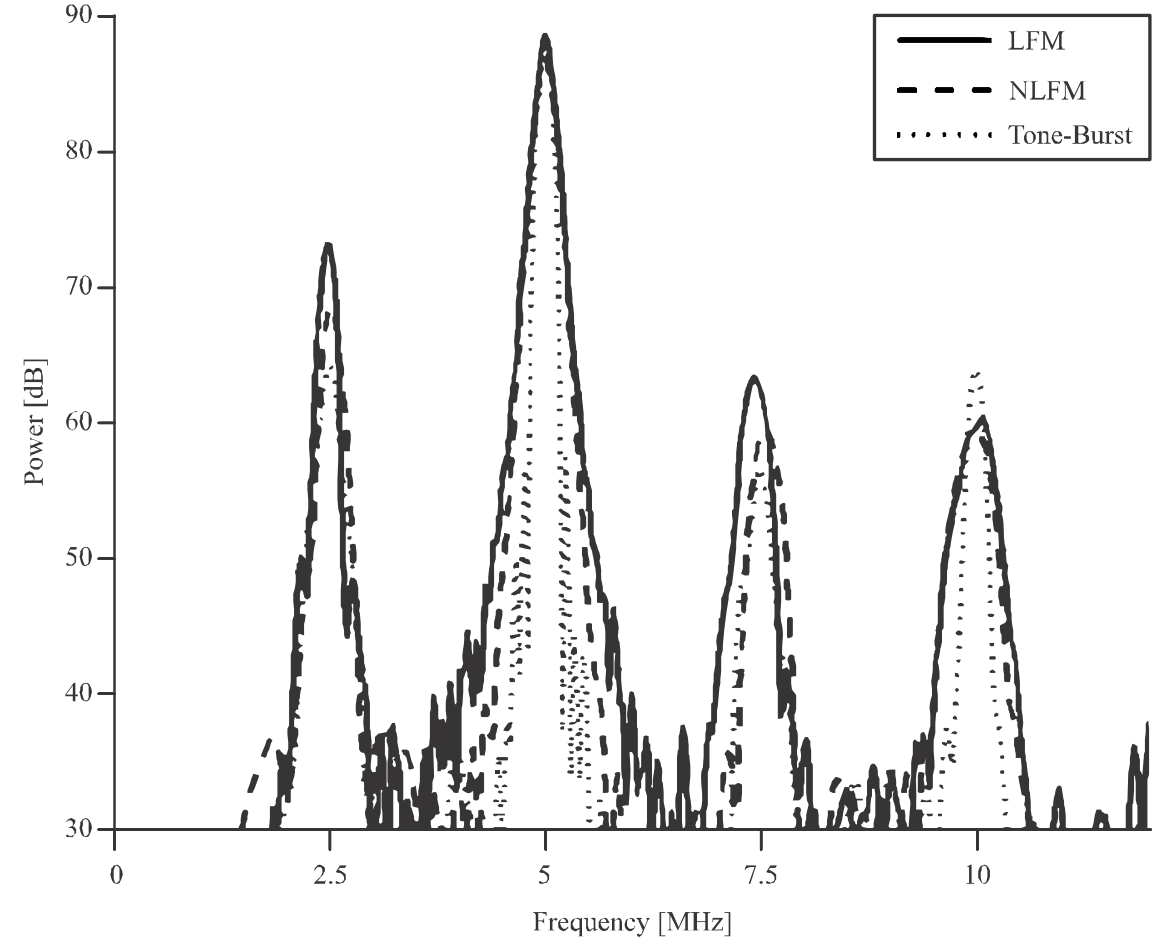

FIG. 2. THE COMPARISON OF POWER SPECTRA OF NLFM, LFM, AND TONE-BURST SIGNALS SCATTERED FROM A COATED MICROBUBBLE WITH A RADIUS OF A $1.7 \mu \mathrm{m}$ AT THE PEAK PRESSURE OF $50 \mathrm{KPa}$

Mehran University Research Journal of Engineering \& Technology, Volume 36, No. 1, January, 2017 [p-ISSN: 0254-7821, e-ISSN: 2413-7219] 
The power spectra comparison of the NLFM signals scattered from a contrast microbubble are shown in Fig. 3. The NLFM signals of 10, 20 and $40 \%$ bandwidth were used as excitation. It is observed that the peak magnitude of the subharmonic component is reducing by increasing the bandwidth of applied signal. For the NLFM signal with a bandwidth of $10 \%$, the subharmonic response is $1.5 \mathrm{~dB}$ higher than the subharmonic response of $20 \%$ bandwidth signal. Similarly, the magnitude of the subharmonic component of $10 \%$ bandwidth signal is $5.5 \mathrm{~dB}$ higher compared to the subharmonic component of the $40 \%$ bandwidth NLFM signal.

The power spectra of the signals scattered from the microbubble with a radius of $1.7 \mu \mathrm{m}$ are shown in Fig. 4 . The up sweep and down sweep NLFM signals with fractional bandwidths of 10,20 , and $40 \%$ were used as excitations. It is shown that the nonlinear harmonic components of the scattered signals are overlapped due to the increase bandwidth of the excitation signal. The overlapping of the harmonic components is clearly visible for $40 \%$ NLFM signal. In imaging applications, the subharmonic component of $40 \%$ bandwidth provides better axial resolution after pulse compression. The pulse compression can be performed with a subharmonic matched filter whose center frequency and bandwidth parameters are half that of the excitation signal. However, partial overlapping of the subharmonic component will make the signal decoding difficult and may reduce the CTR after pulse compression $[31,32,33]$.

It is shown that the subharmonic response from contrast microbubbles is different for up and down sweeps of the NLFM signals. In the case of NLFM signal with a $20 \%$ fractional bandwidth, the subharmonic response of down sweep signal is $2 \mathrm{~dB}$ higher than the up sweep NLFM signal. Similarly, in the case of NLFM signal of $40 \%$ fractional bandwidth, the subharmonic response of down sweep signal is $5 \mathrm{~dB}$ higher than the up sweep NLFM signal. However, for the NLFM signal of $10 \%$ fractional bandwidth, no major difference is found in both up sweep and down sweep due to the use of narrow bandwidth signals.

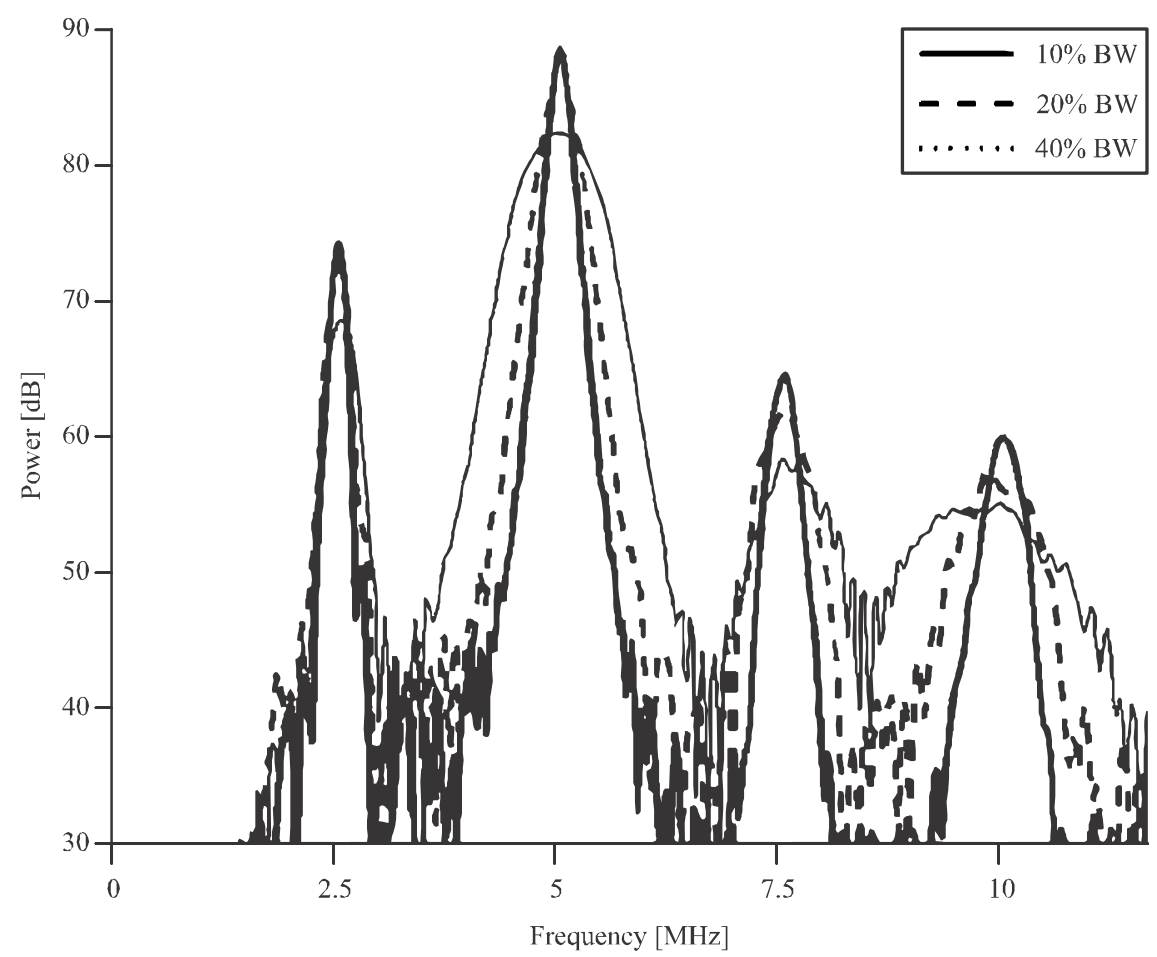

FIG. 3. THE COMPARISON OF NLFM SIGNALS POWER SPECTRA WITH FRACTIONAL BANDWIDTHS OF 10, 20 AND 40\% 
The comparison of the power spectra of NLFM and LFM signals scattered from the contrast microbubble are shown in Fig. 5. The NLFM and LFM with bandwidths of 10,20 , and $40 \%$ were used as excitation signals. It is observed for the same bandwidth signals that the subharmonic response from contrast microbubbles is always higher for NLFM signals compared to the LFM signals.
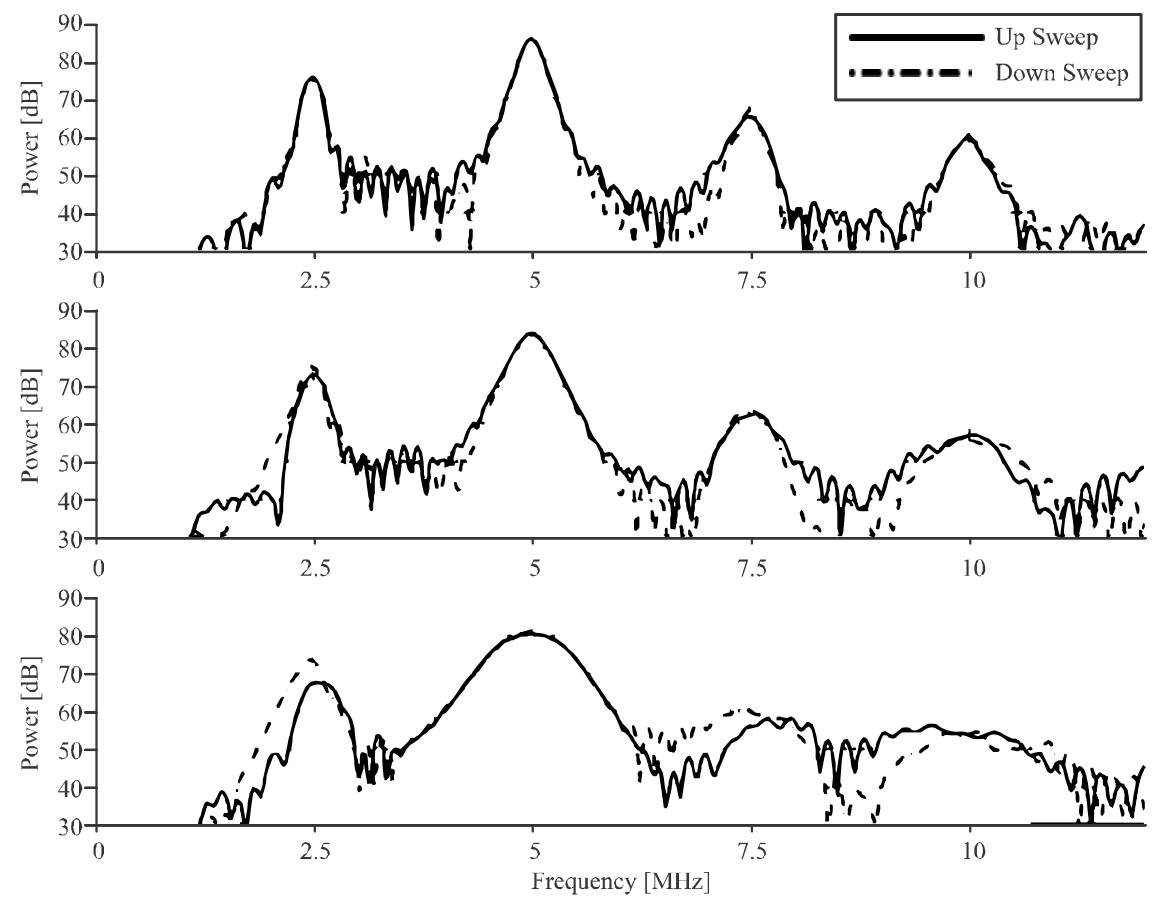

FIG. 4. THE COMPARISON OF SCATTERED POWER SPECTRA FOR THE UP AND DOWN SWEEPS NLFM SIGNALS OF 10\% BANDWIDTH (TOP), WITH A 20\% BANDWIDTH (MIDDLE), AND WITH A 40\% BANDWIDTH (BOTTOM)
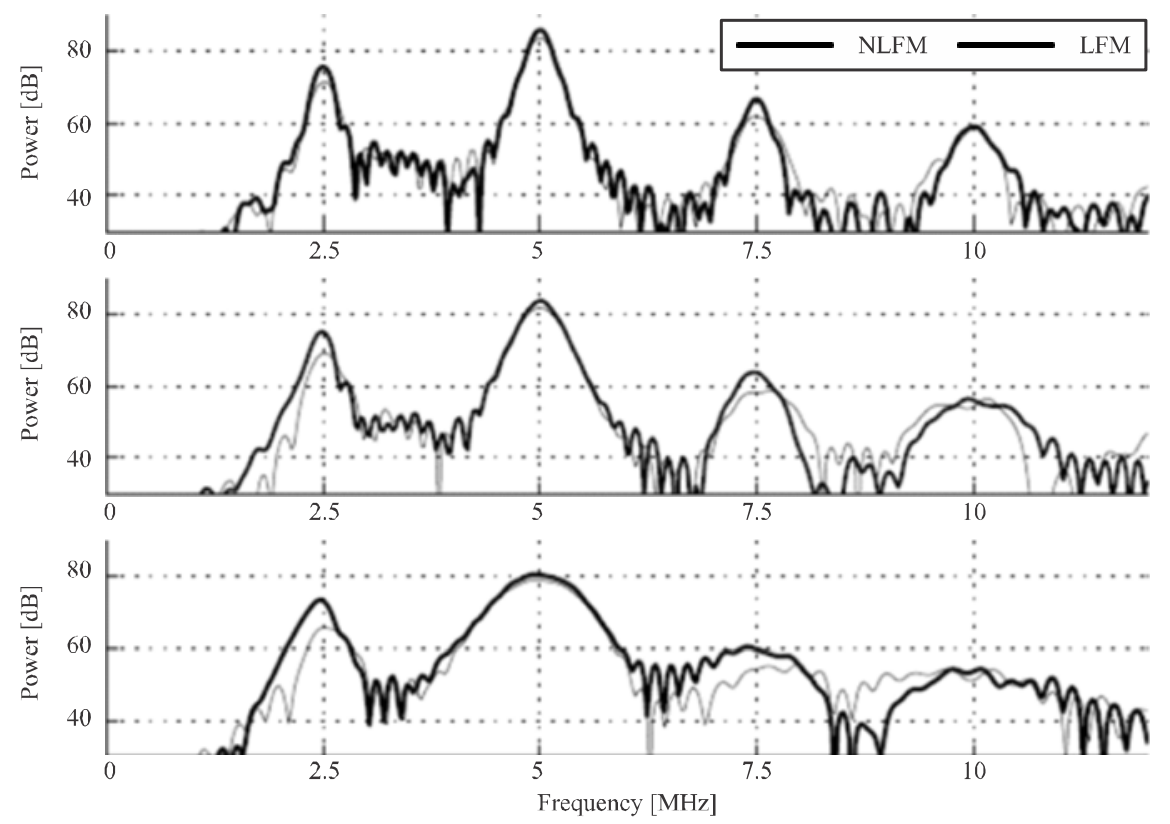

FIG. 5. THE POWER SPECTRA OF THE NLFM AND REFERENCE LFM SIGNALS SCATTERED FROM CONTRAST MICROBUBBLES WITH 10\% BANDWIDTH (TOP), 20\% BANDWIDTH (MIDDLE), AND 40\% BANDWIDTH (BOTTOM)

Mehran University Research Journal of Engineering \& Technology, Volume 36, No. 1, January, 2017 [p-ISSN: 0254-7821, e-ISSN: 2413-7219] 


\section{CONCLUSION}

The subharmonic response from contrast microbubbles can be enhance by using NLFM signals. Ultrasound subharmonic imaging with NLFM signals potentially improve the CTR, SNR and microbubbles detection present in the blood without increasing the peak excitation pressure. The subharmonic frequency component has always higher magnitude for the NLFM signal when compared with the reference tone-burst and LFM signals. It is also observed that increasing the signal bandwidth will reduce the magnitude of the subharmonic component. However, increase bandwidth will improve the axial resolution and hence suitable for subharmonic imaging.

\section{ACKNOWLEDGEMENTS}

Authors are highly grateful to Dr. Steven Freear, University of Leeds, UK, who guided us throughout this work. Authors are also thankful to Mehran University of Engineering \& Technology, Jamshoro, Sindh, Pakistan, for providing all necessary facilities for this research.

\section{REFERENCES}

[1] DeJong, N., Frinking, P.J.A., Bouakaz, A., and Cate, F.J.T., "Detection Procedures of Ultrasound Contrast Agents", Ultrasonics, Volume 38, No. 1-8, pp. 87-92, 2000 .

[2] DeJong, N., Emmer, M., van Wamel, A., and Versluis, M., "Ultrasonic Characterization of Ultrasound Contrast Agents", Medical and Biological Engineering and Computing, Volume 47, No. 8, pp. 861-873, 2009.

[3] Tranquart, F., Grenier, N., Eder, V., and Pourcelot, L., "Clinical Use of Ultrasound Tissue Harmonic Imaging", Ultrasound in Medicine \& Biology, Volume 25, No. 6, pp. 889-894, 1999.

[4] Duck, F.A., "Nonlinear Acoustics in Diagnostic Ultrasound", Ultrasoundin Medicine \& Biology, Volume 28, No. 1, pp. 1-18, 2002.
[5] Krishna, P.D., Shankar, P.M., and Newhouse, V.L., "Subharmonic Generation from Ultrasonic Contrast Agents", Physics in Medicine \& Biology, Volume 44, No. 3, pp. 681-694, 1999.

[6] Forsberg, F., Shi, W.T., and Goldberg, B.B., "Subharmonic Imaging of Contrast Agents", Ultrasonics, Volume 38, No. 1-8, pp. 93-98, 2000.

[7] Shankar, P.M., Krishna, P.D., and Newhouse, V.L., "Advantages of Subharmonic Over Second Harmonic Backscatter for Contrast-to-Tissue Echo Enhancement", Ultrasound in Medicine \& Biology, Volume 24, No. 3, pp. 395-399, 1998.

[8] Eller, A., and Flynn, H.G., "Generation of Subharmonics of Order One Half by Bubbles in a Sound Field", The Journal of the Acoustical Society of America, Volume 46, No. 3B, pp. 722-727, 1969.

[9] Andersen, K.S., and Jensen, J.A., "Impact of Acoustic Pressure on Ambient Pressure Estimation Using Ultrasound Contrast Agent", Ultrasonics, Volume 50, No. 2, pp. 294-299, 2010.

[10] Biagi, E., Breschi, L., Vannacci, E., and Masotti, L., "Subharmonic Emissions from Microbubbles: Effect of the Driving Pulse Shape", IEEE Transactions on Ultrasonics, Ferroelectrics and Frequency Control, Volume 53, No. 11, pp. 2174-2182, 2006.

[11] DeJong, N., Emmer, M., Chin, C.T., Bouakaz, A., Mastik, F., Lohse, D., and Versluis, M., "CompressionOnly Behavior of Phospholipid-Coated Contrast Bubbles", Ultrasound in Medicine \& Biology, Volume 33, No. 4, pp. 653-656, 2007.

[12] Forsberg, F., Dave, J., Halldorsdottir, V., Leodore, L., Lin, F., Hall, A., and Thomenius, K., "Applying RealTime Noninvasive Pressure Estimation Obtained from Subharmonic Contrast Microbubble Signals", IEEE Symposium on Ultrasonics, pp. 1694-1697, 2008.

[13] Goertz, D., Frijlink, M., Tempel, D., Krams, R., DeJong, N., and Van derSteen, A., "Subharmonic Contrast Intravascular Ultrasound", IEEE Symposium on Ultrasonics, pp. 1065-1068, 2007. 
[14] Forsberg, F., Piccoli, C., Ro, R., Lipcan, K., Merton, D., Liu, J., Soparawala, R., Shi, W.T., and Hall, A., "Contrast Enhanced Subharmonic Breast Imaging: Work in Progress", IEEE Symposium on Ultrasonics, Volume 1, pp. 625-628, 2005.

Forsberg, F., Liu, J.B., Shi, W.T., Ro, R., Lipcan, K.J., Deng, X., and Hall, A. L., "In Vivo Perfusion Estimation Using Subharmonic Contrast Microbubble Signals", Journal of Ultrasound Medical, Volume 25, No. 1, pp. 15-21, 2006.

[16] Simpson, D., Chin, C.T., and Burns, P., "Pulse Inversion Doppler: A New Method for Detecting Nonlinear Echoes from Microbubble Contrast Agents", IEEE Transactions on Ultrasonics, Ferroelectrics and Frequency Control, Volume 46, No. 2, pp. 372-382, 1999.

[17] Brock-Fisher, G., Poland, M., and Rafter, P., "Means for Increasing Sensitivity in Nonlinear Ultrasound Imaging Systems", US Patent, No. 5577505, November, 1996.

[18] Eckersley, R.J., Chin, C.T., and Burns, P.N., "Optimizing Phase and Amplitude Modulation Schemes for Imaging Microbubble Contrast Agents at Low Acoustic Power", Ultrasound in Medicine \& Biology, Volume 31, No. 2, pp. 213-219, 2005.

[19] Emmer, M., Vos, H., Versluis, M., and DeJong, N., "Radial Modulation of Single Microbubbles", IEEE Transactions on Ultrasonics, Ferroelectrics and Frequency Control, Volume 56, No. 11, pp. 2370-2379, 2009.

[20] Bouakaz, A., Van der Meer, S., Versluis, M., and DeJong, N., "Chirpreversal Ultrasound Contrast Imaging", IEEE Symposium on Ultrasonics, pp. 224-227, 2006.

[21] Misaridis, T., and Jensen, J., "Use of Modulated Excitation Signals in Medical Ultrasound. Part-I: Basic Concepts and Expected Benefits", IEEE Transactions on Ultrasonics, Ferroelectrics and Frequency Control, Volume 52, No. 2, pp. 177-191, 2005.
[22] Gran, F., and Jensen, J., "Designing Waveforms for Temporal Encoding Using a Frequency Sampling Method", IEEE Transactions on Ultrasonics, Ferroelectrics and Frequency Control, Volume 54, No. 10, pp. 2070-2081, 2007.

[23] Pollakowski, M., and Ermert, H., "Chirp Signal Matching and Signal Power Optimization in Pulse-Echo Mode Ultrasonic Nondestructive Testing", IEEE Transactions on Ultrasonics, Ferroelectrics and Frequency Control, Volume 41, No. 5, pp. 655-659, 1994.

[24] Arif, M., Cowell, D.M.J., and Freear, S., "Pulse Compression of Harmonic Chirp Signals Using the Fractional Fourier Transform”, Journal of Ultrasound in Medicine and Biology, Volume 36, No. 6, pp. 949-956, 2010.

[25] Collins, T., and Atkins, P., "Nonlinear Frequency Modulation Chirps for Active Sonar", IEE Proceedings on Radar, Sonar and Navigation, Volume 146, No. 6, pp. 312-316, 1999.

[26] Marmottant, P., Van der Meer, S., Emmer, M., Versluis, M., de Jong, N., Hilgenfeldt, S., and Lohse, D., "A Model for Large Amplitude Oscillations of Coated Bubbles Accounting for Buckling and Rupture", The Journal of the Acoustical Society of America, Volume 118, No. 6, pp. 3499-3505, 2005.

Gorce, J.M., Arditi, M., and Schneider, M., "Influence of Bubble Size Distribution on the Echogenicity of Ultrasound Contrast Agents: A Study of Sonovue”, Investigative Radiology, Volume 35, No. 11, pp. 661-671, 2000.

[28] Hilgenfeldt, S., Lohse, D., and Zomack, M., "Response of Bubbles to Diagnostic Ultrasound: A Unifying Theoretical Approach", The European Physical Journal B - Condensed Matter and Complex Systems, Volume 4, pp. 247-255, 1998.

[29] Tu, J., Guan, J., Qiu, Y., and Matula, T.J., "Estimating the Shell Parameters of Sonovue ${ }^{\circledR}$ Microbubbles Using Light Scattering", The Journal of the Acoustical Society of America, Volume 126, No. 6, pp. 2954-2962, 2009. 
[30] Arif, M., "Ultrasound Harmonic Imaging using Chirp Coded Excitation", Ph.D. Thesis, School of Electronic \& Electrical Engineering, University of Leeds, UK, 2010 .

[31] Zhang, D., Gong, Y., Gong, X., Liu, Z., Tan, K., and Zheng, H., "Enhancement of Subharmonic Emission from Encapsulated Microbubbles by Using a Chirp Excitation Technique", Physics in Medicine and Biology, Volume 52, No. 18, pp. 5531-5544, 2007.
[32]

Harput, S.M.J., Cowell, D.M.J., and Freear, S., "Contrast-Enhanced Ultrasound Imaging with Chirps: Signal Processing and Pulse Compression” IEEE Symposium on Ultrasonics, pp. 1-4, 2015.

[33] Eisenbrey, J., Sridharan, A., Ji-bin, L., and Forsberg, F., "Recent Experiences and Advances in ContrastEnhanced Subharmonic Ultrasound", Biomedical Research International, ID 640397, pp. 1-6, 2015. 